\title{
EXPLORING TO LEARN WINNING STRATEGY
}

\author{
Samuel Lee \\ SFHS \\ Sunnyvale, California, USA
}

\begin{abstract}
This paper presents a novel algorithm " $U C B$ "” (called "UCB star") to make "intelligent" decisions, enhancing the winning chances within games through the utilization of a well-known tree search algorithm, "Monte Carlo Tree Search (MCTS)". In the typical setting of a MCTS, exploration and exploitation are well balanced to propose "optimal" decisions, given the game status of a board. This happens through the use of the "Upper Confidence Bound (UCB)."

In this paper, a new form of $U C B$ is formulated by taking into account the "posterior" of the parameters, allowing "bias" to be minimized from the observations.

Based on the experiments this paper performed, our $U C B^{*}$ has shown statistically significance of its effectiveness, compared to a traditional $U C B$ algorithm within the MCTS.
\end{abstract}

\section{KEYWORDS}

Board Game, MCTS, Game Strategy, A.I.

\section{INTRODUCTION}

In the past decades, games, ranging from board games, such as Go, to real time strategy (RTS) games, like StarCraft, have been an interesting topic to explore for both research communities and entertainment industries (Bourg and Seemann 2004; Ontanon et al. 2013). Finding "the best" strategy given a state of a game is often considered a challenging task due to the complexity behind most of games. "Go" is a game with such complexity, requiring a space complexity of $10^{171}$ space complexity and tree complexity of $10^{360}$ (Robson 1983). Due to the copious amount of calculations needed, it is not feasible for an A.I. agent to consider all possible movements in a given state. Thus, heuristics and other machine learning algorithms are often utilized (Bouzy and Cazenave 2001; Wang, Wang, and Gao 2017).

MCTS is one of the well-known methods; it is an algorithm used to find the "optima" action by taking random movements in an action space and building them into a tree structure. An example of this is illustrated in Figure 1.

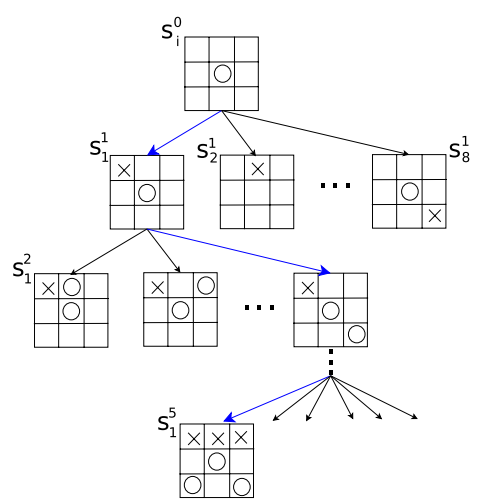

Figure 1. A tree with possible moves given a starting state. Blue arrows are the path of sequential movements 
Note that the figure is based on a well-known simple board game, Tic-Tac-Toc. Each level in Figure 1 shows the possible movements given a state defined in the upper level (known as the parent node).

MCTS is applicable to many different types of games (Sievers and Helmert 2015), particularly ones with decision making processes. The overview of MCTS's process is very straightforward; it is essentially decomposed into four steps - 1) Selection; 2) Expansion; 3) Simulation; and 4) Backpropagation. (The details are found in a survey article (Browne et al. 2012).) For each iteration in building a search tree subject to a constraint (e.g. budget of computations), the first action is to select a "most promising" next movement that balances exploration and exploitation. Note that "exploitation" is to look for the best decision based on learned examples so far, while "exploration" is to try a new probing that has not been known to the space of the learned examples. $S^{1}$ is the example of an action from starting state $S^{0}$, where $S_{m}^{1}$ denotes a state with $\mathrm{m}^{\text {th }}$ possible movement in $l$ level spawned from $(l-1)^{\text {th }}$ parent node. Among possible movements $S^{1}$ at level $l$, where $\mathrm{i} \in\{1 \ldots \mathrm{m}\}$, an "optimal" movement is selected based on a function that quantifies each action w.r.t. the likelihood of winning. Upper Confidence Bound (UCBI) (Auer, Cesa-Bianchi, and Fischer 2002) is an example of such functions quantifying "optimality" by balancing exploitation in the first term and exploration in the second term in Eq. (1). The details of explanations are followed in the next section.

$$
U C B 1\left(S_{i}^{l-1}, S_{j}^{l}\right)=\frac{w(j)}{n(j)}+C \times \sqrt{\frac{2 \ln n\left(f_{j}\right)}{n(j)}}
$$

\section{SMOOTHED UPPER CONFIDENCE BOUNDS: $U C B^{*}$}

In Eq. (1), the first term $w(j) / n(j)$ is interpreted as an estimator for winnings - Bernoulli $n(j)$ trials with $w(j)$ winnings observed. For each trial $x_{t} \in\{0,1\}$, the maximum likelihood estimator $\theta$ is calculated as

$\left[\Sigma\left(I\left(x_{t}==1\right)\right)\right] / N$ by maximizing the likelihood $\Pi_{t=1} \theta^{x_{t}}(1-\theta)^{\left(1-x_{t}\right)}$, which corresponds to $w(j) / n(j)$ in Eq. (1). Note that $\mathrm{I}(\mathrm{c})$ is an indicator function that returns 1 when condition $c$ meets true. By extending the likelihood with prior over $\theta$, the posterior mean, $E\left[\theta \mid w(j), n(j), \alpha_{0}, \alpha_{l}\right]$ is

$$
E\left[\theta \mid w(j), n(j), \alpha_{0}, \alpha_{1}\right]=\frac{w(j)+\alpha_{1}}{n(j)+\alpha_{0}+\alpha_{1}}
$$

Substituting the first term in Eq. (1) with the posterior mean derived in Eq. (2) formulates a new $U C B$, which is smoothed by consider the conjugate prior beta distribution $B\left(\alpha_{0}, \alpha_{1}\right)$.

\section{EXPERIMENTS}

In this section, I report experimental results to show the effectiveness of $U C B^{*}$.

Figure 2 shows statistical significance as $U C B{ }^{*}$ outperforms $U C B 1$ at a p-value of 0.05 from cumulative winning rate results for over 50 runs, where each run is a game played with 2 for $\alpha_{0}$ and 5 for $\alpha_{1}$ and 10 as the simulation budget in a typical MCTS.

In order to see the consistency of the robustness of $U C B{ }^{*}$ over $U C B 1$, more experiments are performed in Figure 3, showing the win rate differences between $U C B^{*}$ and $U C B 1$ through 700 runs, where each run is the cumulative win rate from 50 games played. The areas on the right side of the blue line indicates the winning chances of $U C B$ * over $U C B 1$. 


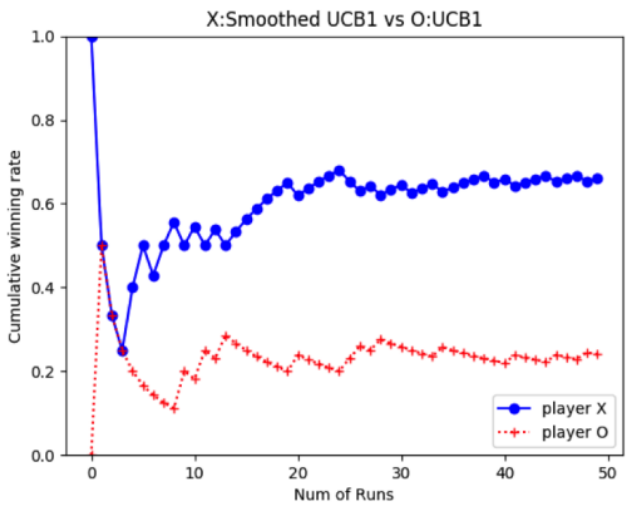

Figure 2. Cumulative win rates by increasing the number of runs to $50 . \mathrm{Y}$ axis shows the cumulative win rate to each run in the $\mathrm{X}$ axis

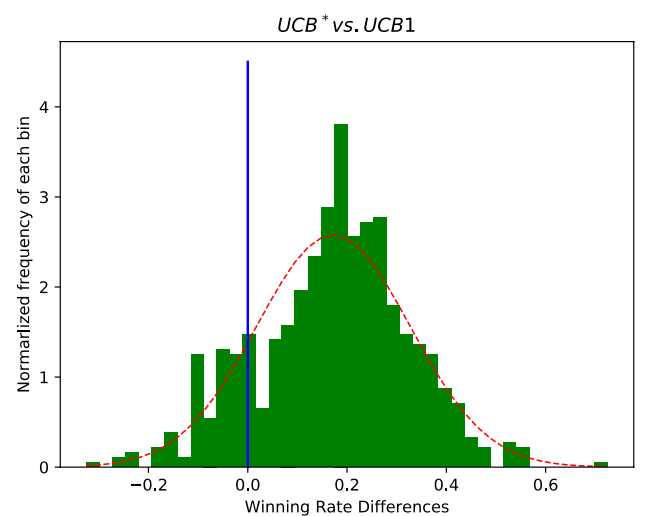

Figure 3. Normalized winning rates in histogram after 700 runs, where each run is the cumulative winning rate from 50 games played

\section{CONCLUSION}

In this paper, a new formulation $\mathrm{UCB}^{*}$ is proposed, and its performance has been proven effectively through the statistical significance over many simulations. For future work, I will investigate 1) how to tune the hyperparameters -- $\alpha_{0}, \alpha_{1}$-- for automatic learning; 2) how to balance exploration and exploitation for better effectiveness as well as faster decisions; and 3) applying the $\mathrm{UCB}^{*}$ to many other use cases.

\section{ACKNOWLEDGEMENT}

I appreciate valuable comments from the anonymous reviewers, which provides many insights for the future endeavors. 


\section{REFERENCES}

Auer, P.; Cesa-Bianchi, N.; and Fischer, P. 2002. Finite-time analysis of the multiarmed bandit problem. Mach. Learn. 47(2-3):235-256.

Bourg, M., and Seemann, G. 2004. AI for Game Developers. O’Reilly Media, Inc.

Bouzy, B., and Cazenave, T. 2001. Computer go: An ai oriented survey. Artificial Intelligence 132(1):39 - 103.

Browne, C.; Powley, E.; Whitehouse, D.; Lucas, S.; Cowl- ing, P. I.; Tavener, S.; Perez, D.; Samothrakis, S.; Colton, S.; and et al. 2012. A survey of monte carlo tree search methods. IEEE T-CIAI.

Ontanon, S.; Synnaeve, G.; Uriarte, A.; Richoux, F.; Churchill, D.; and Preuss, M. 2013. A survey of real-time strategy game ai research and competition in starcraft. IEEE T-CIAIG 5(4):293-311.

Robson, J. M. 1983. The complexity of go. Congress, 413-417.

Sievers, S., and Helmert, M. 2015. A doppelkopf player based on uct. In Holldobler, S.; Penaloza, R.; and Rudolph, S.,eds., KI 2015: Advances in Artificial Intelligence, 151- 165.

Wang, J. W. W.; Wang, R.; and Gao, W. 2017. Beyond monte carlo tree search: Playing go with deep alternative neural network and long-term evaluation. CoRR abs/1706.04052. 